\title{
Recent Developments in the Application of Electromagnetic Waves Propagated in the Earth-Ionosphere Waveguide in Low Latitudes to the Study of 2-Dimensional Earth Models
}

\author{
Ebun ONI and J. A. SOGADE \\ Department of Physics, University of Ibadan, Ibadan, Oyo State, Nigeria
}

(Received January 8, 1992; Accepted February 20, 1992)

\begin{abstract}
Recently we have been trying to appropriately model the spectrum of electromagnetic waves propagated in the Earth-Ionosphere waveguide in low latitudes. We shall present the latest models of such fields and the results of their interactions with 2-dimensional earth models.
\end{abstract}

1. Introduction (Earth-Ionosphere Waveguide in Low Latitudes)

The problem of e.m. wave propagation for a source situated inside the waveguide formed by the Earth and Ionosphere are well documented i.e. see AL'PERT and FLIGEL (1970) and others.

The chief excitation mechanism is by lightning discharge events which generates e.m. waves that are propagated throughout the entire earth via the waveguide. These waves are known as SFERICS. Experimental and theoretical efforts of many scientists have shown that the plane waveguide model works for an observation point very near the source i.e. distance $D \leq 1000 \mathrm{~km}$ to $1500 \mathrm{~km}$ (AL'PERT and FLIGEL, 1970).

One can therefore deduce that near the equatorial region where most of the sources (lightning discharge) exciting low frequency models in the Earth-Ionosphere waveguide, are located, the plane waveguide model will be useful.

In equatorial or low latitude regions (a region of about $\pm 5^{\circ}$ North and South of Dip equator), where there is a non-uniform magnetic field distribution created by a non-uniform current distribution in the Ionosphere, it is important to study the effect of non-uniform magnetic field sources on waveguide propagation.

Many of the naturally excited wave phenomena created by lightning discharge and other sources occur in the extremely or very low frequency ranges.

If all the theoretical problems of these waveguide e.m. fields are solved, they can become natural sources of e.m. fields for the entire study of the conductivity structure of the earth's underground in low latitudes.

In low latitudes the amplitudes of these lightning current pulses are large and can be used to probe deep into the interior of the earth. Recording of large amplitude fields can be made within a period of 4 months every year during the Rainstorm.

However, literature on the Earth-Ionosphere waveguide mode field studies especially due to Wait (from 1957) and Budden (from 1951) and others, show essentially the interaction of such fields with the ionosphere and the results of such studies are applicable for radio 
propagation phenomena in communications etc.

2. Field Equations for the Waveguide Fields and Expressions for Response on Different Earth Structures

The field equations in the waveguide are determined essentially by the nature of the source of origin. Different usual models considered are (a) remote source (located far from observation point) (b) uniform line source at height $h$ above the plane earth surface (c) line source with variations along $x$-direction at height $h$ above earth's surface, (d) dipole source over a plane or spherical earth surface etc.

For the sake of simplicity we shall consider a remote source giving rise to plane electromagnetic waves incident obliquely on the earth's surface. The response curves determined by variations of Reflection coefficient, admittance (for TE-mode), impedance (for TMmode) with frequency are found to be given by equivalent formulas for the cases (a) to (c) above.

Thus, the typical reflection coefficient for such an incident wave from the ionosphere surface (homogeneous half-space model of ionosphere) may be approximately given as

$$
R=\frac{n^{2} \sin \theta-\left(n^{2}-\cos ^{2} \theta\right)^{1 / 2}}{n^{2} \sin \theta+\left(n^{2}-\cos ^{2} \theta\right)^{1 / 2}}
$$

which is Fresnel's reflection coefficient. $n$ is the refractive index of the ionosphere expressed approximately as

$$
n \approx 1-\frac{i N e^{2}}{\varepsilon_{0} m \omega v}=1-i \sigma / \varepsilon_{0} \omega
$$

for frequency $f \leqslant 500 \mathrm{kHz}$. $\sigma$ is the conductivity of ionosphere, $\omega$ the angular frequency, and $\varepsilon_{0}$ the dielectricpermitivity of free space.

Different models of the earth (and ionosphere) structure are possible namely 1-D structural model (conductivity variation along $x$-direction only) 2-D structures (conductivity variation along $x$ and $y$ directions) and 3-D structures (conductivity variations along $x, y$ and $z$-directions).

\subsection{1-D model of the earth (reflection coefficient)}

The typical electric field equation for a uniform plane wave is $E_{n y}=E_{0}\left(T_{n e}^{-U_{m n} z}+\right.$ $\left.R_{n e}^{+U_{m n} z}\right) \mathrm{e}^{-i \lambda x}$ where $T_{n}, R_{n}$ are transmission and reflection coefficients respectively. The reflection coefficient on the earth surface modelled as 1-D structure of $N$-layers (see Fig. 1) (see WAIT (1961)) is given by 


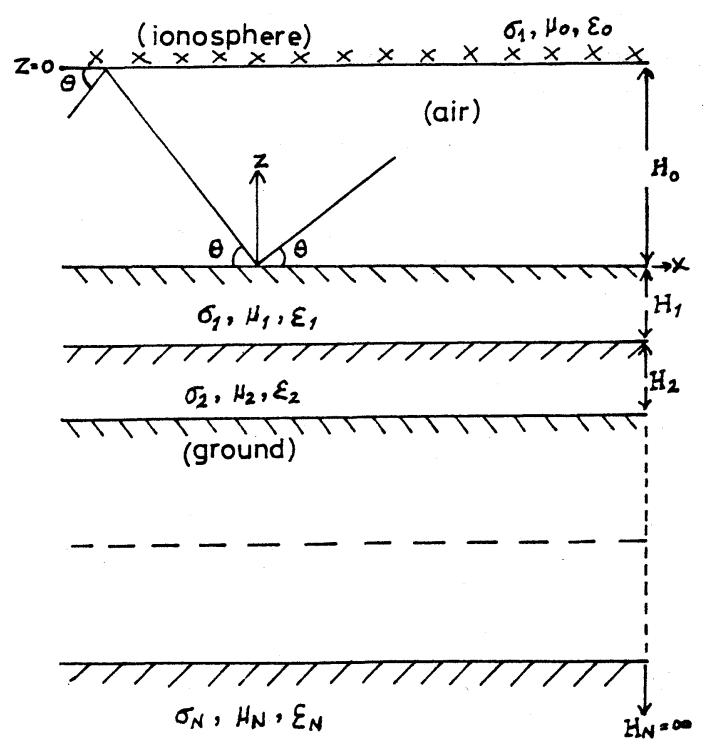

Fig. 1. Schematic of 1-D-N layered model.

$$
R_{g}=\frac{N_{0}-Y_{1}}{N_{0}+Y_{1}} \quad(\mathrm{TE}-\text { mode })
$$

where

$$
Y_{n}=N_{n} \frac{\left(Y_{n+1}+N \tanh U_{m n} H_{n}\right)}{\left(N_{n}+Y_{n+1} \tanh U_{m n} H n\right)} \quad\left(Y_{n}=\text { admittance }\right)
$$

and

$$
N_{n}=\frac{U_{m n}}{i \mu u w} \text { and } U_{m n}=\left[Y_{n}^{2}-\bar{Y}_{0}^{2}\right]^{1 / 2}
$$

For waveguide mode propagation the mode equation $R \cdot R_{g}=\exp \left[2 i\left(K H_{0} \sin \theta-n \pi\right)\right]$ is satisfied, where $H_{0}$ is the height of the ionosphere-base above the earth's surface.

Since there is only a certain prescribed range of values of $\theta$ for the waveguide fields, then there are also only certain allowed values of $R$ and $R_{g}$ for each frequency.

It is interesting to draw an algorithm that solves iteractively for a constant angle $\theta$ from a trial value which satisfies the mode equation and to establish consequent values of $R$ and $R_{g}$, thus $Y_{1}$ for different values of frequencies $(f)$ or angular frequency $(w)$. 


\subsection{2-D models of the earth}

The requisite change will be in the appropriate expression of $R_{g}$ for 2-D Earth Structures. This is achieved by imposing the usual boundary conditions of continuity of magnetic and electric fields across all interfaces. The considered model are shown as case (a) in Fig. 2(a) and (b) in Fig. 2(b).

The appropriate expression that replaces $R_{g}$ for the model in Fig. 2(a) is deduced after application of the following boundary conditions across the interfaces at $z=0, z=z_{1}$, and $x=$ 0 i.e.

$$
\begin{array}{ll}
E_{0 y}=\left.E_{1 y}\right|_{z=0} ; & H_{0 x}=\left.H_{1 x}\right|_{z=0} \quad x \leq x_{0} \\
E_{0 y}=\left.E_{2 y}\right|_{z=0} ; & H_{0 x}=\left.H_{2 y}\right|_{z=0} \quad x \geq x_{0} \\
E_{1 y}=\left.E_{2 y}\right|_{x=x_{0}} ; & \mu_{1} H_{1 n}=\left.\mu_{2} H_{2 x}\right|_{x=x_{0}} .
\end{array}
$$

The basic electric and magnetic field equations

$$
\begin{aligned}
& E_{n y}=E_{0}\left(T_{n e}^{-U_{m n} z}+R_{n e}^{U_{m n} z}\right) \mathrm{e}^{-i \lambda x}, \\
& H_{n y}=E_{0}\left(T_{n e}^{-U_{m n} z}-R_{n e}^{U_{m n} z}\right) \mathrm{e}^{-i \lambda x}
\end{aligned}
$$

have to be modified to take cognisance of the reflecting capability of the interface at $x=x_{0}$ i.e.

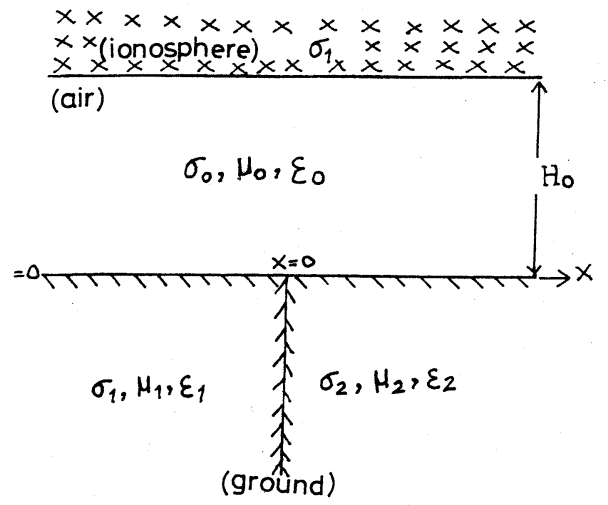

(a)

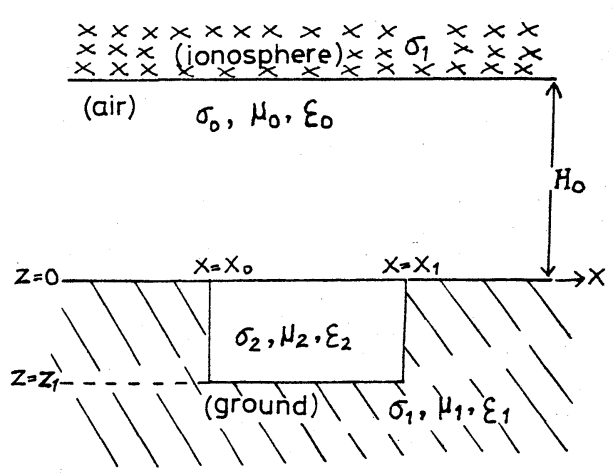

(b)

Fig. 2. (a) Schematic of 2 D-half-space model. (b) Schematic of 2 D-layered model. 


$$
\begin{aligned}
& E_{n y}=E_{0}\left(T_{n z} \mathrm{e}^{-U_{m n} z}+R_{n z} \mathrm{e}^{U_{m n} z}\right)\left(T_{n x} \mathrm{e}^{-i \lambda x}+R_{n x} \mathrm{e}^{+i \lambda x}\right), \\
& H_{n y}=N_{n} E_{0}\left(T_{n z} \mathrm{e}^{-U_{m n} z}+R_{n z} \mathrm{e}^{U_{m n} z}\right)\left(T_{n x} \mathrm{e}^{-i \lambda n}+R_{n x} \mathrm{e}^{+i \lambda x}\right),
\end{aligned}
$$

Application of Eqs. (2) in (1) leads to the following matrix equations.

$$
\left|\begin{array}{cccccc}
1, & 0, & -1, & \mathrm{e}^{-2 \bar{Y}_{0} x}, & 0, & 0 \\
-N_{0}, & 0, & -N_{1}, & -N_{1} \mathrm{e}^{+2 \bar{Y}_{0} x}, & 0, & 0 \\
0, & 1, & 0, & 0, & -1, & -\mathrm{e}^{+2 \bar{Y}_{0} x} \\
0, & -N_{0}, & 0, & 0, & -N_{2}, & -N_{2} \mathrm{e}^{+2 \bar{Y}_{0} x} \\
0, & 0, & \mathrm{e}^{-\bar{Y}_{0} x}, & \mathrm{e}^{+\bar{Y}_{1} x_{0},} & -\mathrm{e}^{-\bar{Y}_{2} x_{0}}, & -\mathrm{e}^{+\bar{Y}_{2} x_{0}} \\
0, & 0, & \mu_{1} N_{1} \mathrm{e}^{-\vec{Y}_{1} x_{0}}, & \mu_{1} N_{1} \mathrm{e}^{\bar{Y}_{0} x_{0}}, & \mu_{2} N_{2} \mathrm{e}^{-\bar{Y}_{2} x_{0}}, & \mu_{2} N_{2} \mathrm{e}^{-\bar{Y}_{2} x_{0}}
\end{array}\right| \times\left|\begin{array}{c}
R_{0 z}^{1} \\
R_{0 z}^{2} \\
T_{1 z} \\
T_{1 z} T_{1 x} \\
T_{2 z} T_{2 x} \\
T_{2 z} R_{2 x}
\end{array}\right|=\left|\begin{array}{c}
-1 \\
-N_{0} \\
-1 \\
-N_{0} \\
0 \\
0
\end{array}\right|
$$

Thus, $R_{g}=R_{0 z}^{1}$ when the reflection coefficient is derived for $x \leq x_{0}$ and $R_{g}=R_{0 z}^{2}$ for $x>x_{0}$.

Solution of the above matrix equation is accomplished by the LU-decomposition + backsubstitution technique.

For the model in Fig. 2(b) similar expression like in (3) are deduced from which $R_{g}$ can be determined.

The admittance $Y_{1}$ is deduced from the expression $Y_{1}=\left(R_{g}-N_{0}\right) /\left(R_{g}+N_{0}\right)$ in each case.

3. Determination of Response Characteristics and Curves Showing Interactions

A typical algorithm for determining each $R_{g}$ and $Y_{1}$ for chosen frequencies $(w)$ is deduced from the following

Step 1: Choose frequency $w$

Step 2: Choose a trial value of $\theta$ (real) $=\theta_{\text {old }}$.

Step 3: Calculate $R, R_{\mathrm{g}}$ from earlier formulas for $\theta$ and $w$ (using chosen values)

Step 4: Solve for $\theta$ (or $\sin \theta)$ using, $R R_{g}=\exp \left[2 i\left(K H_{0} \sin \theta-n \pi\right)\right]$

Step 5: Test for self-consistency i.e. is $\theta_{\text {old }}<>\approx \theta$ new or not

Step 6: Stop if answer is positive (i.e. if $\theta_{\text {old }} \approx \theta_{\text {new }}$ ) and then

Step 7: Calculate $Y_{1}$ from $Y_{1}=\left(R_{g}-N_{0}\right) /\left(R_{g}+N_{0}\right)$

Step 8: Store final $R_{g}, Y_{1}$

Step 9: If answer to test 5 is negative (i.e. $\theta_{\text {old }}<>\theta_{\text {new }}$ ) then

Step 10: Repeat procedure with the new values of $\theta$ i.e. go to step 3.

Step 11: Repeat for other frequencies $(w)$ i.e. go back to step 1.

The calculated values of $R_{g}, Y_{1}$ from a computer implementation of the above algorithm are plotted against a parameter $D\left(=\mu w H_{0}\right)$.

Note that other practical considerations outside the computer program are needed to ensure convergent solution i.e. appropriate choice of $\sigma_{\mathrm{i}}$ (ionosphere conductivities) etc.

Curves showing the interaction for 3-layered 1-D Earth model $(N=3$ in Fig. 1$)$ are shown 


\section{REFLECT.COEFF.(R) VERSUS PARA.(D)}

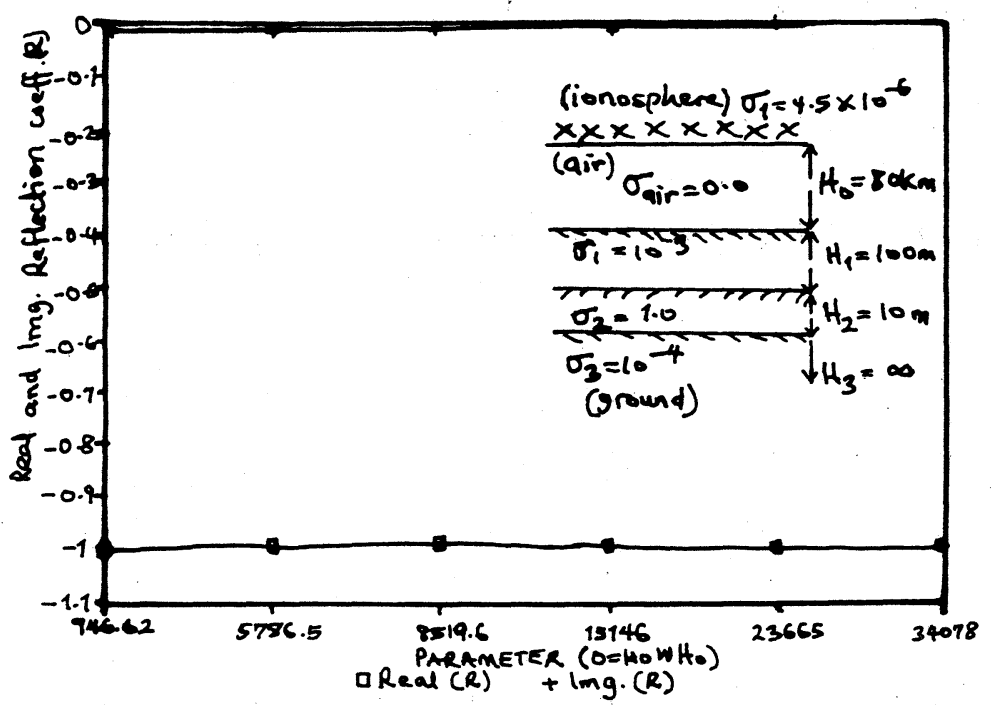

Fig. 3. Variation of reflection coefficient with parameter $D\left(=\mu_{0} w H_{0}\right) 1-\mathrm{D}$ structure.

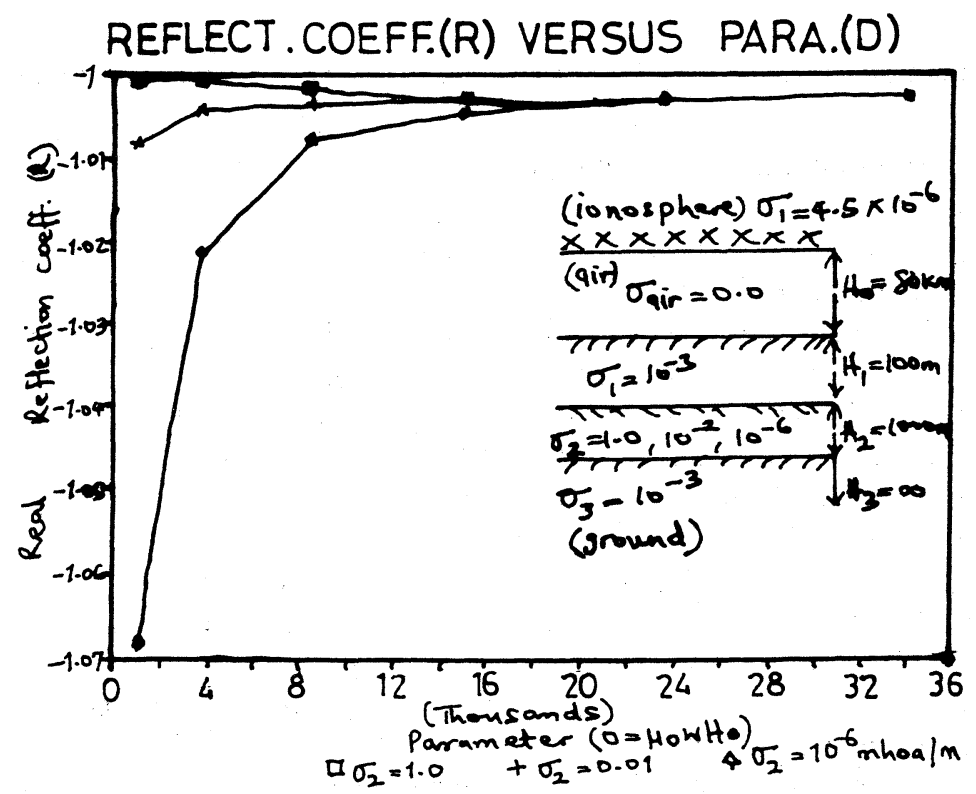

Fig. 4. Variation of reflection coefficient with parameter $D\left(=\mu_{0} w H_{0}\right)$ 1-D structure. 


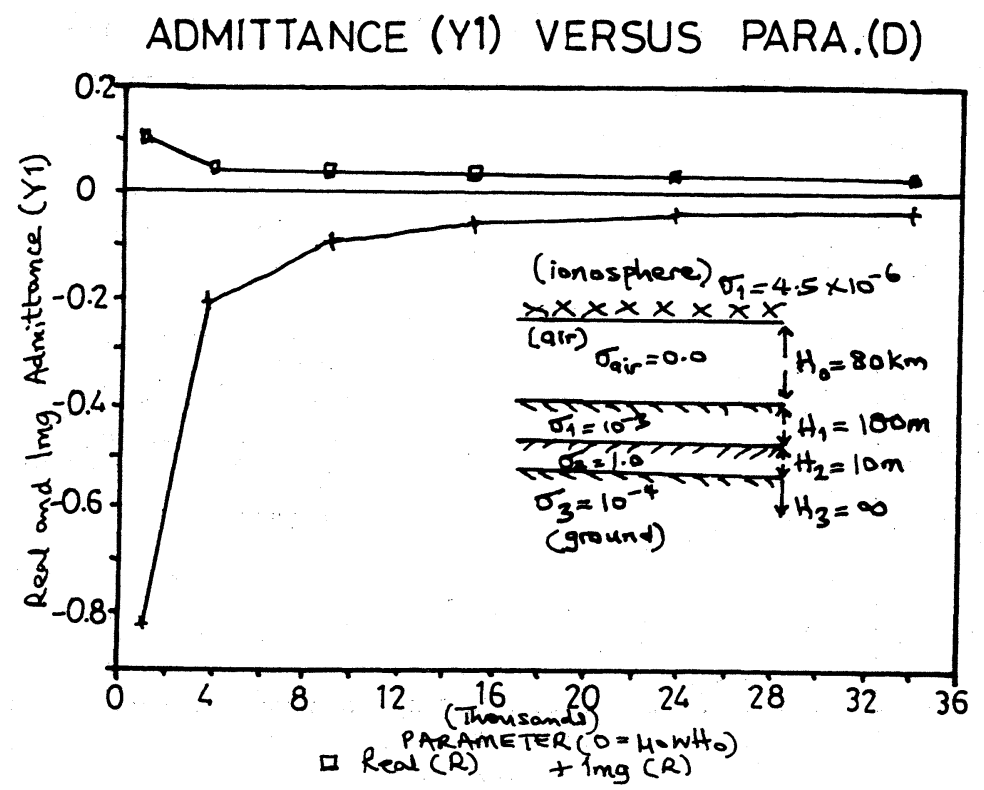

Fig. 5. Variation of admittance with parameter $D\left(=\mu_{0} w H_{0}\right)$ 1-D structure.

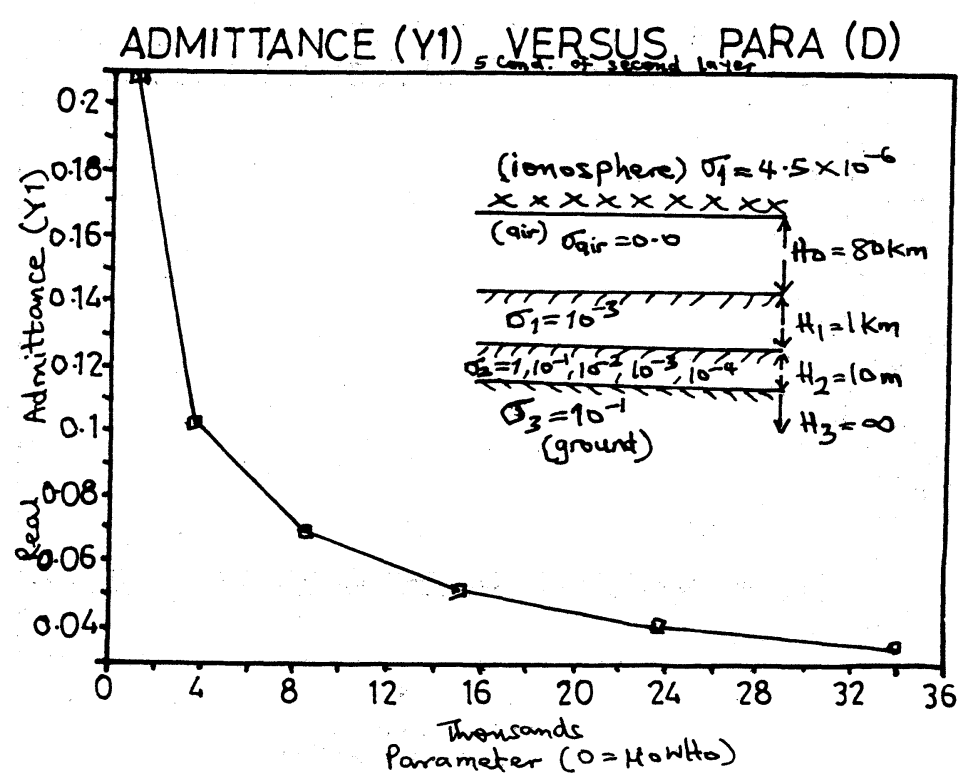

Fig. 6. Variation of admittance with parameter $D\left(=\mu_{0} w H_{0}\right)$ 1-D structure. 


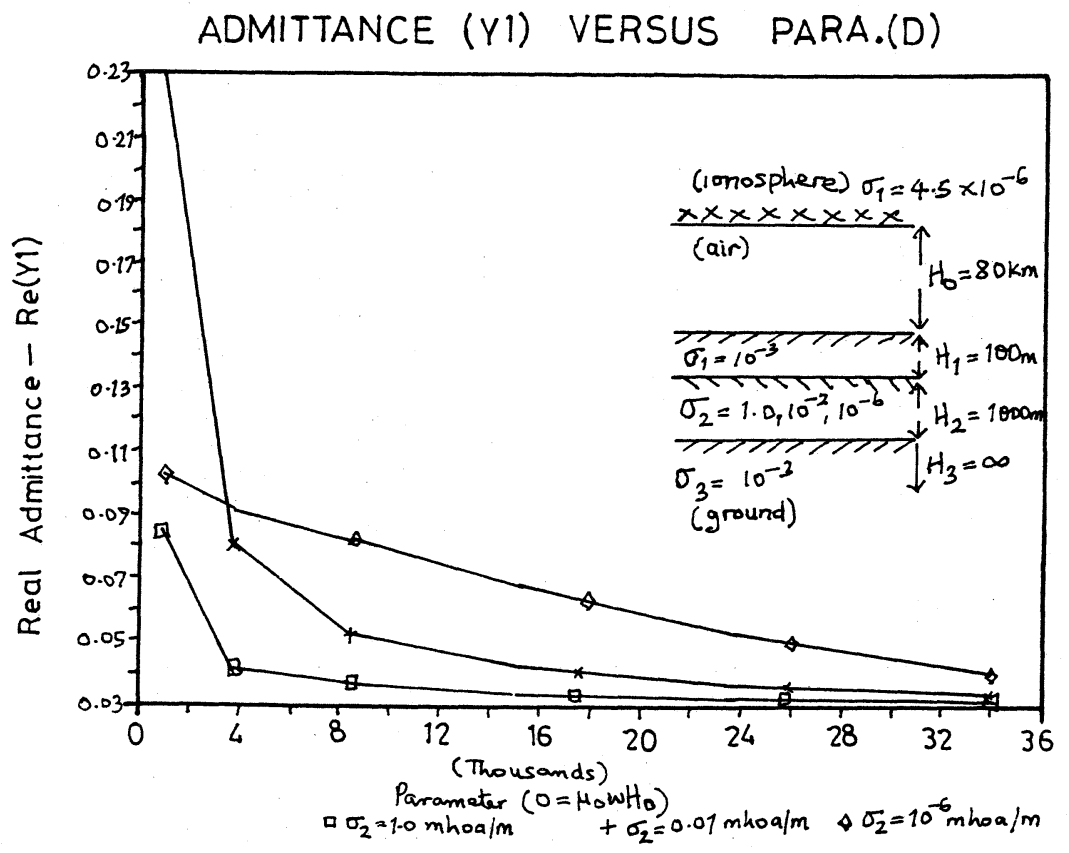

Fig. 7. Variation of admittance with parameter $D\left(=\mu_{0} w H_{0}\right) 1-\mathrm{D}$ structure.

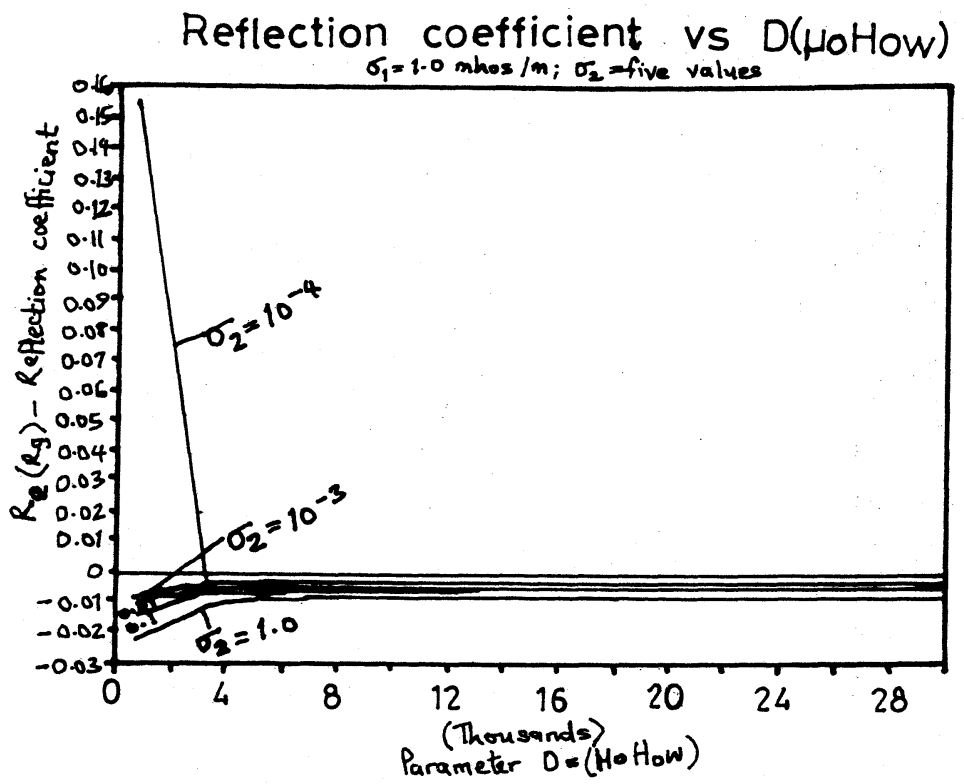

Fig. 8. Variation of reflection coefficient with parameter $D\left(=\mu_{0} w H_{0}\right) 2 \mathrm{D}$ half-space model. $x_{0}=0.0 \mathrm{~m}, x=100 \mathrm{~m}$. 


\section{Admittance (Y1) vs $D$ ( HoHow)}

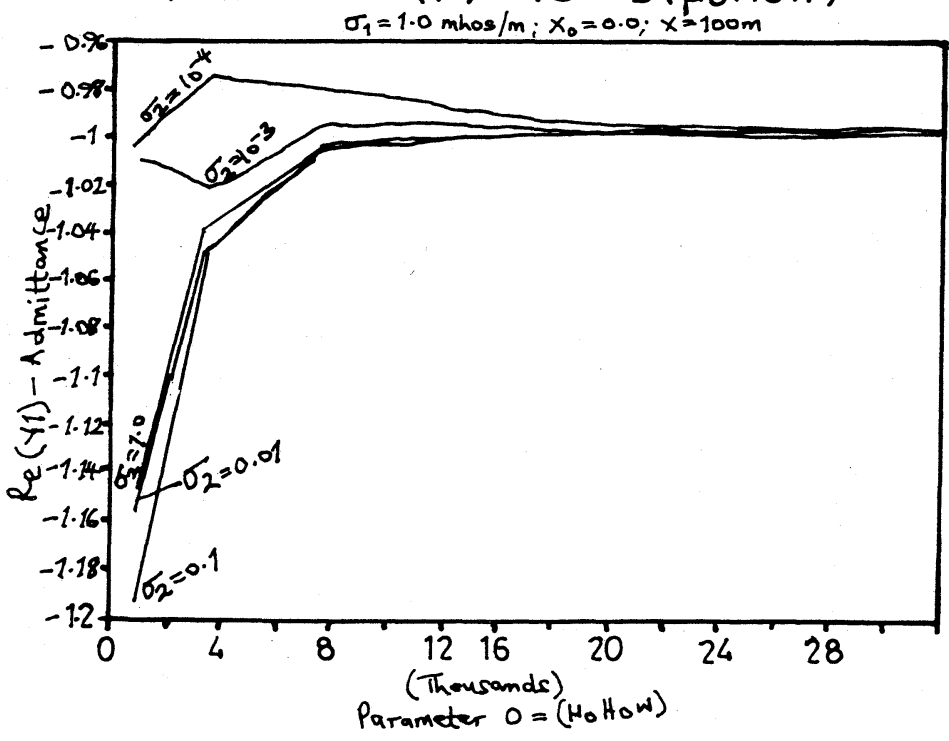

Fig. 9. Variation of admittance with parameter $D\left(=\mu_{0} w H_{0}\right) 2 \mathrm{D}$-half-space model.

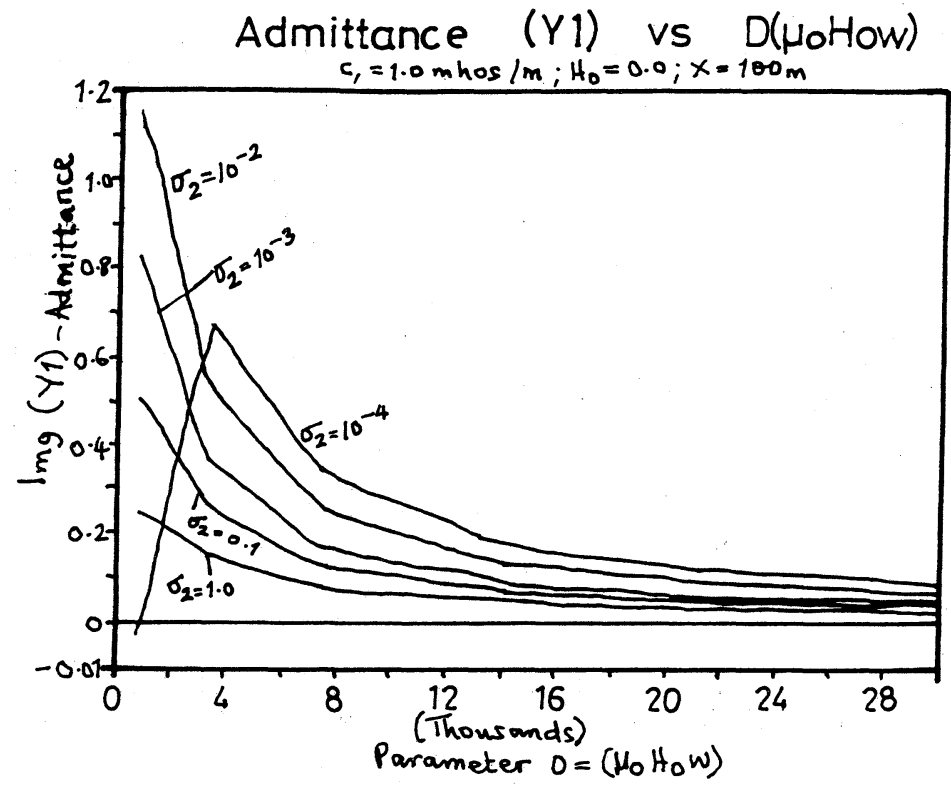

Fig. 10. Variation of admittance with parameter $D\left(=\mu_{0} w H_{0}\right) 2 \mathrm{D}$-half-space model. 


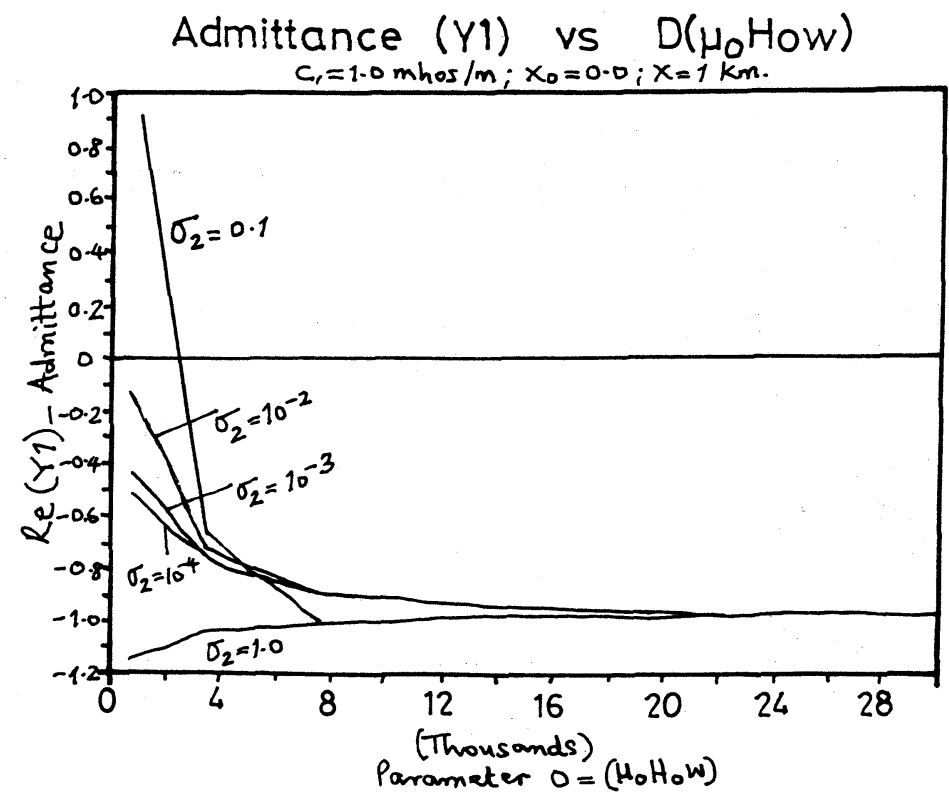

Fig. 11. Variation of admittance with parameter $D\left(=\mu_{0} w H_{0}\right) 2 \mathrm{D}$-half-space model.

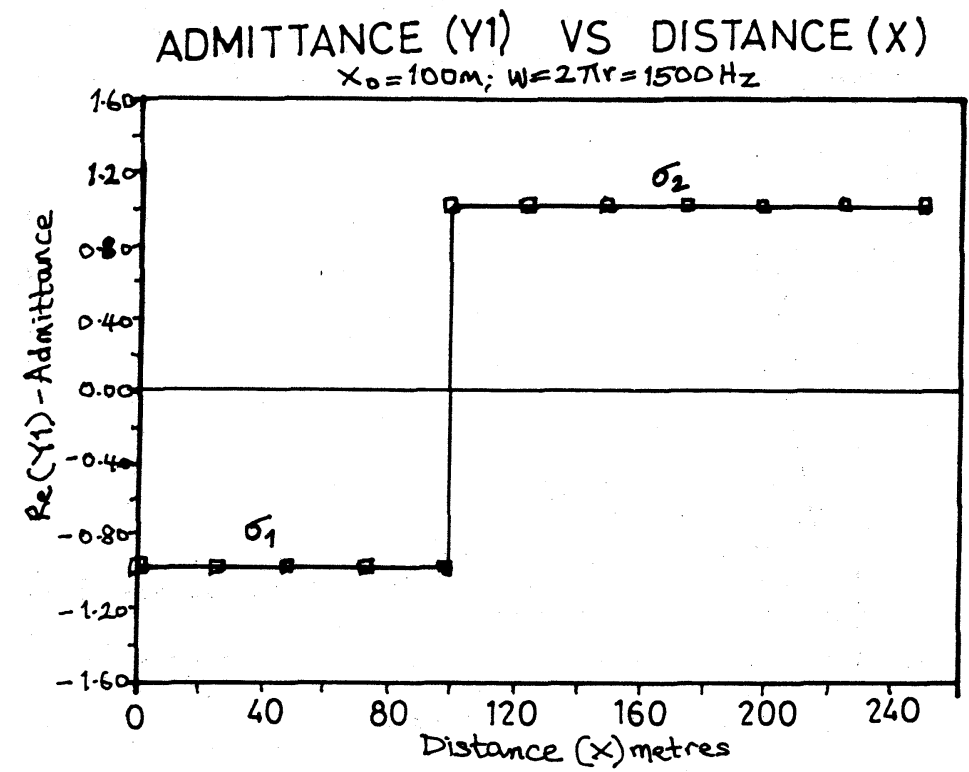

Fig. 12. Variation of real (admittance) with parameter $D\left(=\mu_{0} w H_{0}\right) 2 \mathrm{D}$-half-space model. 


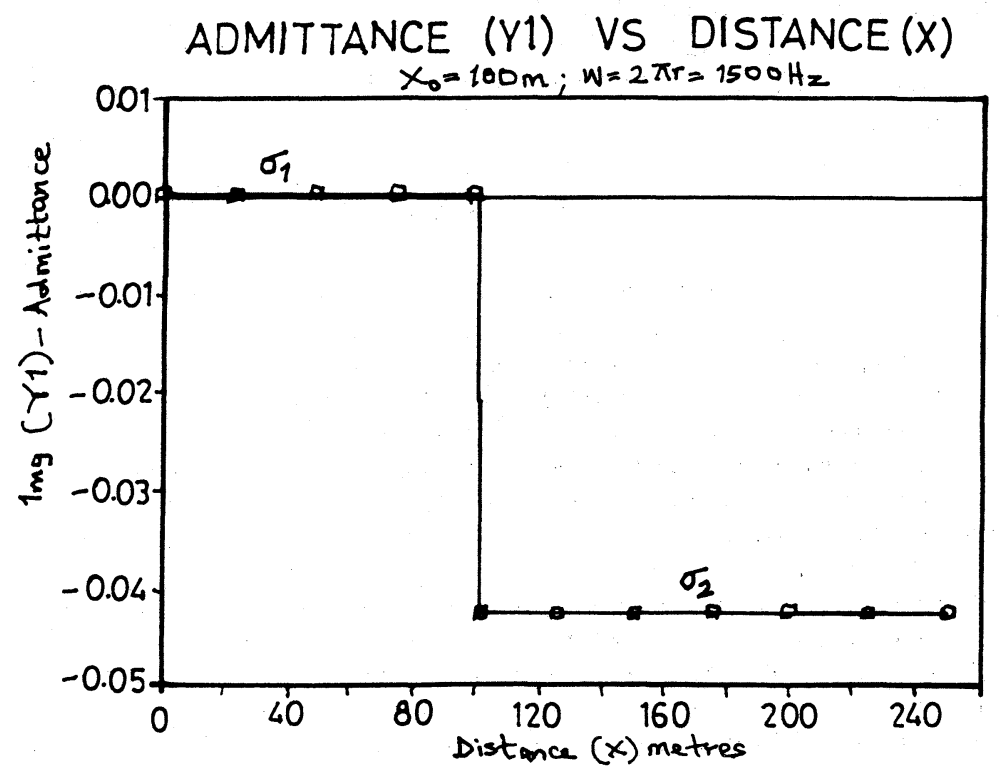

Fig. 13. Variation of Imag. (admittance) with parameter $D\left(=\mu_{0} w H_{0}\right) 2 \mathrm{D}$ half-space model.

in the Figs. 3 to $7 \sigma_{\mathrm{i}}=4.5 \times 10^{-6} \mathrm{mhos} / \mathrm{m}$ is used (explanation follows from the figures).

It is enough to show only the results of case (a) in the 2-D model. Since this will be enough to highlight the essential features of the method. Such results are shown in Figs. 8 to 13.

\section{Concluding Remarks}

(a) From the results of the modelling we have carried out for 1-D and 2-D Earth Structures, we are convinced that these natural low frequency sources from lightning discharge, are useful for the study of the interior of the earth in low latitudes. What we need now is the design of suitable instrumentation for monitoring these electromagnetic fields during lightning discharge.

(b) There is need to carry out more theoretical modelling in order to deduce more realistic equations which take into account the effect on waveguide propagation, of the non-uniform source fields of the electrojet.

The different models of the Earth considered serve as a basis for future work into more complex and challenging models in 2 and possibly 3-D structures.

Also the ionosphere could be extended into a more realistic number of layers depending on time of day and other ionospheric parameters.

Finally, one can see that more realistic propagation characteristics of e.m. waveguide mode fields used in communications can be derived from these models as necessary when fully developed.

The study is a combination of (a) Ionospheric Physics, (b) Atmospheric Physics, (c) Solid Earth Physics. 
The authors acknowledge with thanks Professor P. Kaikkonen and the entire members of staff of the International Laboratory of Numerical Electromagnetic Modelling (ILONEM), Department of Geophysics, University of Oulu, Oulu, Finland for allowing the use of their computer facilities to carry out some parts of this work.

\section{REFERENCES}

AL'PERT, Ya. L. and D. S. FLIGEL, Propagation of ELF and VLF Waves near the Earth, Consultants Bureau, New York, 1970.

BudDen, K. G., The propagation of a radio, Atmospheric. Phil. Mag., 42, 1-19, 1951a.

BUDDEN, K. G., The reflection of very low frequency radio waves at the surface of a sharply bounded ionosphere with superimposed magnetic field, Phil. Mag., 42, 883-850, $1951 \mathrm{~b}$.

BudDen, K. G., The propagation of a radio atmospheric. II, Phil. Mag., 43, 1179-1200, 1952.

BuDden, K. G., The Waveguide Mode Theory of Wave Propagation, Logos, 1962.

WAIT, J. R., The mode theory of V.L.F. ionospheric propagation for finite ground conductivity, Proc. Inst. Radio Eng., 45, 760-767, 1957a.

WAIT, J. R., The attenuation vs. frequency characteristics of V.L.F. radio waves, Proc. Inst. Eng., 45, 768-771, 1957b.

WAIT, J. R., On the mode theory of V.L.F. ionospheric propagation, Geofisica Pura e applicata, 37, 103-115, 1957c.

WAIT, J. R., Influence of an inhomogenious ground on the propagation of VLF radio waves in the Earth-Ionosphere waveguide, J. Res. Nat. Bur. Std., 09 D(7), 1965.

WAIT, J. R., Electromagnetic Waves in Statified Media, Pergamon, New York, 1970. 